\title{
Parallel Hybrid Trajectory Based Metaheuristics for Real-World Problems
}

\author{
Gabriel Luque \\ Universidad de Málaga, Andalucía Tech \\ E.T.S.I. Informática, Campus Teatinos, \\ 29071 Málaga (España) \\ gabriel@lcc.uma.es
}

\author{
Enrique Alba \\ Universidad de Málaga, Andalucía Tech \\ E.T.S.I. Informática, Campus Teatinos, \\ 29071 Málaga (España) \\ eat@lcc.uma.es
}

\begin{abstract}
This paper proposes a novel algorithm combining path relinking with a set of cooperating trajectory based parallel algorithms to yield a new metaheuristic of enhanced search features. Algorithms based on the exploration of the neighborhood of a single solution, like simulated annealing (SA), have offered accurate results for a large number of realworld problems in the past. Because of their trajectory based nature, some advanced models such as the cooperative one are competitive in academic problems, but still show many limitations in addressing large scale instances. In addition, the field of parallel models for trajectory methods has not deeply been studied yet (at least in comparison with parallel population based models). In this work, we propose a new hybrid algorithm which improves cooperative single solution techniques by using path relinking, allowing both to reduce the global execution time and to improve the efficacy of the method. We applied here this new model using a large benchmark of instances of two real-world NP-hard problems: DNA fragment assembly and QAP problems, with competitive results.
\end{abstract}

Keywords-parallelism; trajectory based metaheuristics; path relinking; QAP; DNA fragment assembly;

\section{INTRODUCTION}

Metaheuristics are general heuristics that provide suboptimal solutions in a reasonable time for various optimization problems [11]. According to the number of solutions they manage during optimization process, they fall into two categories: trajectory based methods and population based techniques. On the one hand, a population based metaheuristic makes use of a randomly generated population of solutions. The initial population is enhanced through a natural evolution process. At each generation of the process, the whole population or a part of the population is replaced by newly generated individuals (often the best ones). On the other hand, a trajectory based algorithm starts with a single initial solution which, at each step of the search, is replaced by another (often better) solution found in its neighborhood.

Although the use of metaheuristics allows to significantly reduce the temporal complexity of the search process, the exploration remains time-consuming for many industrial and engineering problems. In this context, parallelism emerges as a useful strategy to reduce the computational times down to affordable values. The point is that the parallel versions of metaheuristics allows not only to speed up the computations, but also to improve the quality of the provided solutions
[1], [18]. For both trajectory-based and population-based metaheuristics, different parallel models have been proposed in the literature. In general, these parallel models are mostly oriented to study parallel population-based algorithms, but it actually exists a gap in the studies about parallel models for single solution methods from which something could be gained for other researchers.

The focus of this paper is on parallel trajectory-based metaheuristics. Usually, three major parallel models for this kind of algorithms exist: the parallel exploration of the neighborhood, the parallel evaluation of each solution, and the multi-start model. The two first models speed up the execution of the method without changing the semantics of the algorithm in comparison with a sequential exploration. The last one is maybe more interesting from the algorithmic point of view since it can change the behavior of the method with respect to its serial counterpart. The multi-start model lies in launching in parallel several independent or cooperative homo/heterogeneous algorithms. Usually, in its cooperative mode, subalgorithms of the parallel multi-start model exchange information (solutions) during execution and when the target subalgorithm receives a solution, it continues the search using the previous one or the newly received one according to a selection scheme. The problem of this cooperative model is that some interesting information is lost since either the new solution is discarded (it is not chosen by the selection scheme) and no new information is incorporated, or it is accepted and the previous historical information of the subalgorithm is lost.

This work proposes a new parallel yet simple model that extends the cooperative multi-start model to avoid the aforementioned flaw. In this case, when a solution is received, we use the path relinking technique to generate a set of new candidate solutions which combine the information of the new solution and the previous tentative solution of the target subalgorithm. Therefore, the subalgorithm incorporates new information but, at the same time, it keeps information of its own search. The utilization of path relinking opens some design alternatives: which subalgorithms cooperate, which solution of the set generated by path relinking will be selected to continue the search, ... Then, one goal of this paper is to propose a new cooperative scheme and study 
the different design alternatives. Other important objective of this work is the utilization of this parallel model to solve two real-world NP-hard problems: DNA fragment assembly [17] and QAP problems [16].

This paper is organized as follows. The next section (Section II) introduces some background information about parallel trajectory based methods and path relinking. Section III presents our proposed parallel model. Then we describe the experimental design used in this paper. Later, we discuss experimental results in Section V and finally we summarize the conclusions and give some hints on the future work.

\section{BACKGROUND}

In this section, we present some background information about the basic techniques that we use to design our new proposal described in this paper.

\section{A. Trajectory based techniques}

Trajectory based methods, illustrated in Algorithm 1, are single solution-based metaheuristics dedicated to the improvement only one solution in its neighborhood. They start their exploration process from an initial solution randomly generated or provided by another metaheuristic. This solution is then updated during a certain number of steps. At each step, the current solution is replaced by another (often the better) one found in its neighborhood. These methods are mainly characterized by: an internal memory storing the state of the search, a strategy for the selection of the initial solution, a generator of candidate solutions i.e. the neighborhood, and a selection policy of the candidate moves. Three major trajectory based algorithms are largely used: Hill Climbing (HC) [16], Simulated Annealing (SA) [14], and Tabu Search (TS) [12].

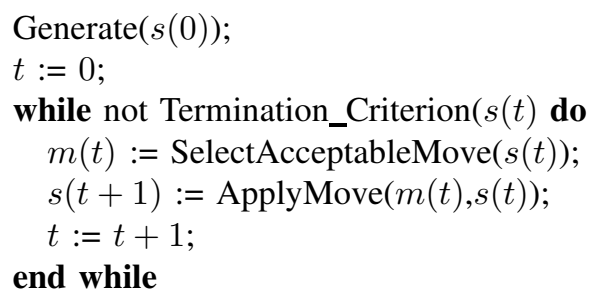

Algorithm 1: Trajectory based technique skeleton pseudocode

Although these technique allows to obtain very accurate results for a large number of problems, some advanced mechanisms have to be used to tackle with the high requirements of the industrial problems. One of these mechanisms is the utilization of parallel models. In the literature are usually identified three major parallel distributed models of this kind of method [2]: the parallel exploration of the neighborhood, the multi-start model, and the parallel evaluation of each solution.
- Parallel multi-start model: The model consists in launching in parallel several independent or cooperative homo/heterogeneous single solution method. Each subalgorithm is often initialized with a different solution. The independent approach is widely exploited because it is natural and easy for the user. In this case, the semantics of the model is the same as the serial execution. That is to say the results obtained with $N$ parallel independent methods is the same as that provided by $N$ algorithms performed in a serial way on a single machine. The parallelism allows to efficiently enhance the robustness of the execution.

In its cooperative mode, subalgorithms of the parallel multi-start model exchange information during execution. Usually that information is a solution.

- Parallel exploration of the neighborhood model: This parallel model is a kind of farmer/worker model allowing to speed up the exploration of the possible moves without changing the semantics of the algorithm in comparison with a sequential exploration. At the beginning of each iteration of the algorithm, the farmer sends the current solution to a pool of workers. Each worker explores some neighboring candidates, and returns back the results to the farmer.

- Parallel evaluation of solution model: The fitness of each solution is evaluated in a parallel centralized way. This kind of parallelism could be efficient if the evaluation function is CPU time-consuming and/or IO intensive.

In the literature, we can found parallel versions of the most popular trajectory based metaheuristics such as parallel SA [9], [4], parallel VNS [19], [8], parallel TS [3], [6], ... But most of them are focused on the application to be solved and they use classical parallel models. The aim of this paper is the parallel model itself, our goal is to provide a new mechanism to build more efficient and accurate parallel solution-based techniques.

The two last models uses the parallel platform to speedup the search procedure but they don't change the behavior of the method. On the contrary, the first parallel model using cooperation modify the dynamics of the technique. In this work, we focus in this kind of methods.

\section{B. Path Relinking}

Path relinking (PR) [13] was originally proposed into the context of scatter search by extension of its basic philosophy. $\mathrm{PR}$ is based on the generation of paths between high quality solutions. This leads to a broader conception of the meaning of creating combinations of solutions. Such combinations may be conceived to arise by generating paths between and beyond selected solutions in neighborhood space, rather than in Euclidean space. This conception is reinforced by the fact that a path between solutions in a neighborhood space will generally yield new solutions that share a significant subset 


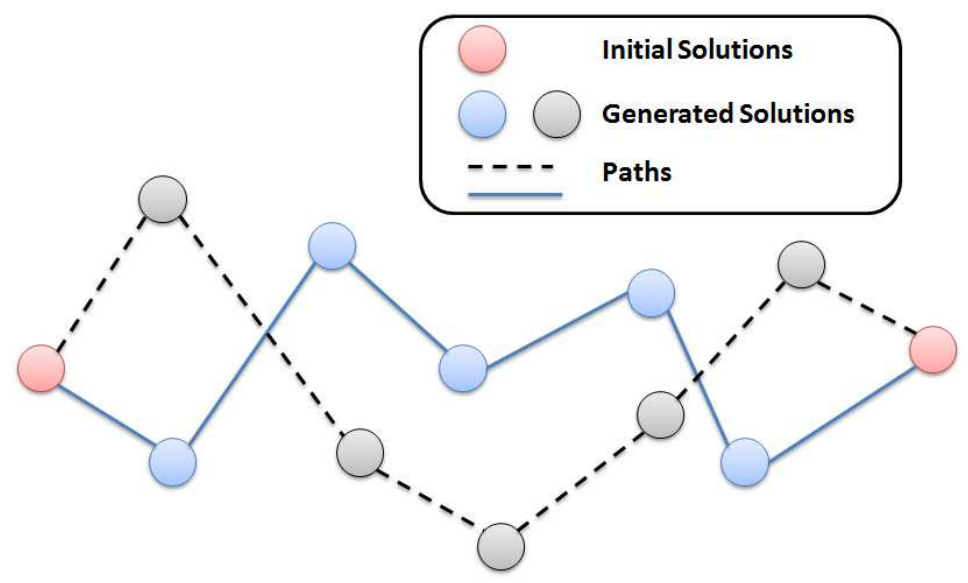

Figure 1. Path relinking scheme.

of attributes contained in the parent solutions, in varying "mixes" according to the path selected and the location on the path that determines the solution currently considered. The character of such paths is easily specified by reference to solution attributes that are added, dropped or otherwise modified by the moves executed in neighborhood space. To generate the desired paths, it is only necessary to select moves that perform the following role: upon starting from an initiating solution, the moves must progressively introduce attributes contributed by a guiding solution (or reduce the distance between attributes of the initiating and guiding solutions). In Figure 1, we can observe the scheme followed for this technique to obtain new solutions.

\section{OUR PRoposed Model}

Our goal is to design a new parallel model for trajectory based metaheuristics which allows to reduce the global execution time but, at the same time, it also improves the efficacy of the exploration of the search space. A number of papers has been devoted to this topic for parallel approaches involving population based methods (some of them also involving trajectory-based ones) but it is not a very studied field for pure parallel trajectory based metaheuristics.

Since we want to improve the efficacy of the resulting parallel algorithm, we focus on the multi-start cooperative paradigm (the other two models do not change the dynamics of the method with respect to the serial version). As discussed in the introduction, a problem in classical approaches of multi-start models for trajectory-based metaheuristics is the lost of information. Indeed, when a subalgorithm receives a solution from other subalgorithm, it has to choose whether it continues the search either with the current one or the newly received one, loosing the stored information in the discarded solution.

We propose a new model in which we do not have to choose between the two solutions, but generate a new solution with the main features of both solutions. With this aim, we can use some mechanisms, similar to the recombination operator of population based method, which combine both solutions [15]. But, in this work, we propose the utilization of a more advanced technique such as path relinking. We run this technique to generate some paths using the current solution and the incoming solution as initial points. The generated path provides the parallel technique of a set of candidate solutions to continue the search, and therefore, a selection scheme is needed to chose one.

Several important design issues arise from the general model proposed in this work:

- Cooperation scheme: it indicates what and how subalgorithms cooperate each other.

- Selection scheme: it refers what solution is selected from the set of candidate ones generated by PR.

For each design feature we have proposed some alternatives. In the previous existing multi-start models, the features of the incoming solution were not very important rather than it fitness value, but now, this issue can provoke an important impact in the search behaviour. Different possible cooperation schema are analyzed here:

- Predefined: in this case, each subalgorithm receives a single solution (the sending island is defined by the topology). Therefore, any subalgorithm only receives a single solution which is combined with the local one.

- Depending of the fitness value (best): in this case, each subalgorithm receives a solution from each subalgorithm which composes the global method. Now, the subalgorithm has to select one solution from this set of candidate solutions, that will be combined with the current one. In this strategy, the selection mechanism is based on the fitness value of the incoming solutions. In this study, we select the solution with the best fitness.

- Depending of the features of the solution (distance): 
as in the previous one, each subalgorithm receives several solutions (one per subalgorithm) and it has to select one. In this case, the selection will be performed by using a genotypic distance (a diversity measure) among the solutions and we select the farthest one. This distance depends on how the solution are represented in the algorithm.

- Random: as in the previous scenarios, each subalgorithm receives several solutions (one per subalgorithm) but in this strategy, random one is selected from all the incoming solutions.

Using one of the these strategies, our proposed technique obtains the second initial solution (the first initial one is the current point of the subalgorithm) and then, we can apply the path relinking to generate a path. That path provides some new candidate solutions and the method has to select one of them to replace the current one and continue the search process. To choose the new solution we also take into account some alternatives:

- Best: the algorithm selects the best solution in the generated path:

$$
\max _{s \in S}(f(s))
$$

where $S$ is a set of solution composed by the points visited during the path generated by $\mathrm{PR}$ and $f$ is the fitness function (assuming the maximization case).

- Most shared information: in this case, the subalgorithm selects the solution sharing more information from initial solutions. To do this, we calculate the genotypic distance among the solutions and then we apply the next equation:

$$
\min _{s \in S}\left(\max \left(\operatorname{dist}\left(s, s^{\prime}\right), \operatorname{dist}\left(s, s^{\prime \prime}\right)\right)\right),
$$

where $S$ is a set of solution composed by the points visited during the path generated by $\mathrm{PR}, s^{\prime}$ and $s^{\prime \prime}$ are the initial solutions and dist is the genotypic distance between two solutions. With this process we simultaneously minimize the distance of the new solution with respect to the two initial ones.

- Random: in this case, the subalgorithm select a random solution from the path without taking into account its quality or any other feature.

In the experimental section we study the behaviour and the performance of each strategy.

\section{EXPERIMENTAL DESIGN}

In this section, we describe the experimental design followed in this work. First, we present the problems and the instances used in the experiments. Later, we explain the algorithm used to test our parallel model. Finally, we present the methodology and parameters used in the experiments.

\section{A. Benchmark}

In order to make more relevant contribution, we have selected a wide set of instances from two very different problems: DNA fragment assembly and QAP.

DNA fragment assembly: In order to determine the function of a specific genes, scientists have learned to read the sequence of nucleotides comprising a DNA sequence in a process called DNA sequencing. To do that, multiple exact copies of the original DNA sequence are made. Each copy is then cut into short fragments at random positions. These small fragments can be read in the laboratory by specialized equipment. After the fragment set is obtained, a traditional assemble approach is followed in this order: overlap, layout, and then consensus. To ensure that enough fragments overlap, the reading of fragments continues until a coverage is satisfied. We give a brief description of each of the three phases, namely overlap, layout, and consensus:

- Overlap Phase - Finding the overlapping fragments. This phase consists of finding the best or longest match between the suffix of one sequence and the prefix of another. In this step, we compare all possible pairs of fragments to determine their similarity. Usually, a dynamic programming algorithm applied to semiglobal alignment is used in this step. The intuition behind finding the pairwise overlap is that fragments with a significant overlap score are very likely next to each other in the target sequence.

- Layout Phase - Finding the order of fragments based on the computed similarity score. This is the most difficult step because it is hard to tell the true overlap due to the several challenges: unknown fragment orientation, base call errors, incomplete coverage, repeated regions, and chimeras and contamination. After the order is determined, the progressive alignment algorithm is applied to combine all the pairwise alignments obtained in the overlap phase.

- Consensus Phase - Deriving the DNA sequence from the layout. The most common technique used in this phase is to apply the majority rule in building the consensus.

From a combinatorial optimization viewpoint, the whole process of constructing of the consensus sequence is similar to that of a tour in the Travelling Salesman Problem (TSP). This is because each fragment would have to be in a specific fragment ordering sequence in order for the formation of a consensus sequence to take place. The main difference between TSP and DNA fragment assembly is that there would not be a proper alignment between the first and the last fragments in the consensus sequence that is comparable to the connection between the first and the last cities in the TSP solution. Therefore, many equivalent solutions to TSP are thus inequivalent in our context. Other important 
difference is that while the ordering is the final solution to TSP, in our case, this ordering is only an intermediate step and several different orderings can produce equivalent results. Other minor differences can be found between both problems due to the challenges described previously (unknown orientation, incomplete coverage, etc.).

To measure the quality of a consensus, we can look at the distribution of the coverage. Coverage at a base position is defined as the number of fragments at that position. It is a measure of the redundancy of the fragment data, and it denotes the number of fragments, on average, in which a given nucleotide in the target DNA is expected to appear. It is computed as the number of bases read from fragments over the length of the target DNA [17].

$$
\text { Coverage }=\frac{\sum_{i=1}^{n} \text { length of the fragment } i}{\text { target sequence length }}
$$

where $n$ is the number of fragments. The higher the coverage, the fewer number of the gaps, and the better the result.

To test and analyze the performance of our algorithm we generated several problem instances with GenFrag [7]. GenFrag takes a known DNA sequence and uses it as a parent strand from which random fragments are generated according to the criteria supplied by the user (mean fragment length and coverage of parent sequence).

We have chosen four sequences from the NCBI web site $^{1}$ : a human apolopoprotein HUMAPOBF, with accession number M15421, which is 10,089 bases long; the complete genome of bacteriophage lambda, with accession number J02459, which is 20k bases long; and the Neurospora crassa (common bread mold) BAC, with accession number BX842596, which is 77,292 bases long.

Table I

INFORMATION OF DATASETS

\begin{tabular}{cccccc}
\hline Parameters & M15421 & Instance & J02459 & BX842596 \\
\hline Coverage & 5 & 7 & 7 & 4 & 7 \\
Fragment length & 398 & 383 & 405 & 708 & 703 \\
Nb. of fragments & 127 & 177 & 352 & 442 & 773 \\
\hline
\end{tabular}

QAP: The Quadratic Assignment Problem (QAP) is a wellknown NP-hard combinatorial optimization problem, which is the core of many real-world optimization problems [10]. QAP models many applications in diverse areas.

Let $P$ be a set of $n$ facilities and $L$ a set of $n$ locations. For each pair of locations $i$ and $j$, an arbitrary distance is specified $r_{i j}$ and for each pair of facilities $p$ and $q$, a flow is specified $w_{p q}$. The QAP consists of assigning to each location in $L$ one facility in $P$ in such manner that the total cost of the assignment is minimized. Each location can only contain one facility and all facilities must be assigned to one

\footnotetext{
${ }^{1}$ http://www.ncbi.nlm.nih.gov/
}

location. For each pair of locations, the cost is calculated as the product of the distance between the locations and the flow associated with the facilities in the locations. The total cost is the sum of all the costs associated with each pair of locations. One solution to this problem is a bijection between $L$ and $P$, that is, $x: L \rightarrow P$ such that $x$ is bijective. Without loss of generality, we can just assume that $L=P=$ $\{1,2, \ldots, n\}$ and each solution $x$ is a permutation in $S_{n}$, the set permutations of $\{1,2, \ldots, n\}$.

The cost function to be minimized can be formally defined as:

$$
f(x)=\sum_{i, j=1}^{n} r_{i, j} \cdot w_{x(i), x(j)}
$$

We chose the five most complex QAP instances of QAPLIB Library ${ }^{2}$. The complexity of the QAP instances is given by their size and autocorrelation length $\ell$ [5]. In consequence, we selected esc128, tho150, tai100b, tai150b, and tai256c instances that are described in Table II.

Table II

THE FIVE MOST COMPLEX INSTANCES OF QAPLIB.

\begin{tabular}{crrrr}
\hline Instance & Size & $\xi$ & $\ell$ & Lowest Known Cost \\
\hline esc128 & 128 & 32 & 32 & 64 \\
tho150 & 150 & 41.19 & 44.174 & $8.13 \mathrm{E}+06$ \\
tai100b & 100 & 35.472 & 39.613 & $1.19 \mathrm{E}+09$ \\
tai150b & 150 & 40.458 & 42.947 & $4.99 \mathrm{E}+08$ \\
tai256c & 256 & 64 & 64 & $4.48 \mathrm{E}+07$ \\
\hline
\end{tabular}

\section{B. Algorithm}

We have used the well-known simulated annealing algorithm to test our parallel model. Simulated annealing (SA) [14] is a stochastic optimization technique, which has its origin in statistical mechanics. It is based upon a cooling procedure used in industry. This procedure heats the material to a high temperature so that it becomes a liquid and the atoms can move relatively freely. The temperature is then slowly lowered so that at each temperature the atoms can move enough to begin adopting the most stable configuration. In principle, if the material is cooled slowly enough, the atoms are able to reach the most stable (optimum) configuration. This smooth cooling process is known as annealing. Algorithm 2 shows a scheme of SA. First at all, the parameter $T$, called the temperature, and the solution, are initialized. The solution $s 1$ is accepted as the new current solution if $\delta=f(s 1)-f(s 0)<0$. Stagnations in local optimum are prevented by accepting also solutions which increase the objective function value with a probability $\exp (-\delta / T)$ if $\delta>0$. This process is repeated several times to obtain good sampling statistics for the current temperature. The number of such iterations is given by the parameter Markov_Chain_length, whose name alludes the fact that the sequence of accepted solutions

\footnotetext{
${ }^{2}$ http: //www. seas. upenn.edu/qaplib/
} 
is a Markov chain (a sequence of states in which each state only depends on the previous one). Then the temperature is decremented and the entire process repeated until a frozen state is achieved at $T_{\min }$. The value of $T$ usually varies from a relatively large value to a small value close to zero.

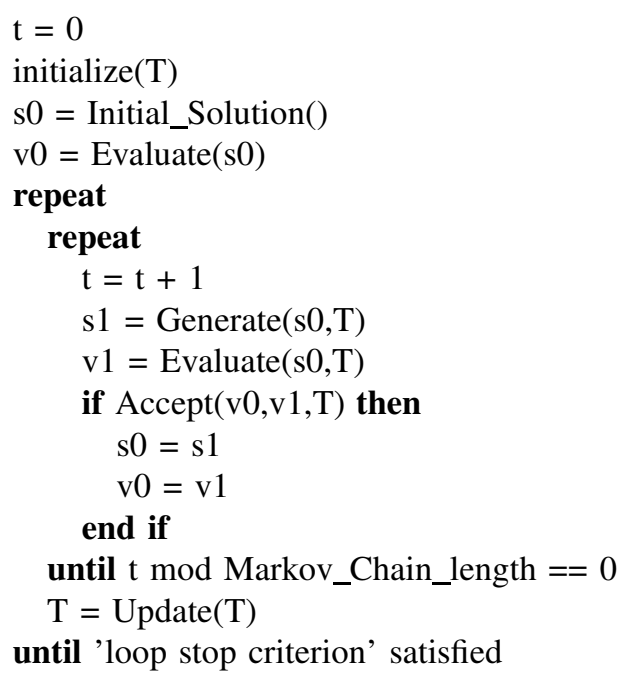

Algorithm 2: Scheme of the Simulated Annealing(SA) Algorithm.

In order to apply SA to solve our problems, we have to define how a solution in the neighborhood is generated (function Generate in Algorithm 2). Since we use an integer permutatio to represent a solution for both problem, we use the standard swap operator (the values of two positions are interchanged) to build a new solution from the current one.

In our parallel approach we run eight independent instances (subalgorithms) of SA which asynchronously cooperate every 50 iterations. As cooperation scheme, we use the four alternatives presented in Section III. When a new solution arrives to the target subalgorithm, the PR method is applied to generate a path. To build this path, first, it analyzes the component which are different between the two initial solutions, and then, each visited point in the path is generated by changing one of these components.

This way of building the path allows us to obtain efficient implementations of the selection scheme (see Section III). For example, we do not need to build the whole path when we want to select a random solution from the path or when we plan to use a solution which maximizes the shared information, since they can be calculated a priori only generating a single solution. However, to obtain the best solution of the path, all the visited points should be generated and evaluated. In order to the reduce the computation cost of this last strategy, we will use partial evaluations instead of a complete evaluation of the solution. This allows to reduce the computational complexity of the evaluation of a solution from $O\left(n^{2}\right)$ (a complete evaluation) to $O(n)$ (when a partial evaluation is performed).

\section{Methodology}

This subsection provides the reader with the details of the experiments performed to evaluate the new parallel model proposed for trajectory-based metaheuristics. We have analyzed 12 different variants (three selection strategies and four cooperation schema). We use the terminology SA_X_Y, where $\mathbf{X}$ is the selection mechanism and $\mathbf{Y}$ is the cooperation strategy. The possible values for $\mathbf{X}$ are: rnd for random, bst for best, and inf for maximizing the shared information scheme. The possible values for $\mathbf{Y}$ are: pre for the predefined topology, bst for the best solution, dst for the strategy based on the distance, and rnd for the random one. We will also compare our proposed model with a parallel version using the multi-start no-cooperative model, also known as independent run model (iSA), and a parallel version using the classical multi-start cooperative model (cSA), in which incoming solutions just replace the current one. In order to perform fair comparisons, the stopping condition is to find the optimal solution.

The experiments have been executed on a Intel Xeon(R) CPU E3-1220 v3 @ 3.10GHz with 16 GB running Ubuntu Linux 14.4. Because of the stochastic nature of the algorithms, we perform 30 independent runs of each test to gather meaningful experimental data and apply statistical confidence metrics to validate our results. First, we use the Kolmogorov-Smirnov test to check whether the data follows a normal distribution or not. If so, then we carry out an ANOVA test to compare the means; otherwise, a KruskalWallis test is used to compare the medians. In each case, a confidence level of $99 \%$ is used.

\section{ANALYSIS OF THE EXPERIMENTS}

In this section we analyze the results of the different variants of our proposed model. First, we study the accuracy of the methods and then, we discuss their computational cost.

\section{A. Accuracy}

Let's first compare the accuracy of the different algorithms. Since there are many different problem instances and analyzing them thoroughly would hinder us from drawing clear conclusions, we have summarized in Table III the results. In this table we only study the accuracy of the techniques. Since the stopping criterion is to find the optimal solution (if it is possible since some variants get stuck in a local one), to measure the accuracy we use the number of instances solved by the method (the algorithm was able to find the optimum). We use two different values: the first one is the number of instances in which the algorithm found the optimal solution in at least one run; and the second one is the number of instances in which the algorithm found the 
optimal solution in robust way (this means the algorithm find the optimum in at least 25 out 30 independent runs). The range of both values is between 0 and 10 ( 5 instances of QAP plus 5 instances of DNA Fragment Assembly)

\begin{tabular}{lccc}
\hline Cooperation scheme & \multicolumn{3}{c}{ Selection scheme } \\
& bst & rnd & inf \\
\hline pre & $7-3$ & $5-1$ & $6-3$ \\
bst & $\mathbf{1 0}-\mathbf{1 0}$ & $\mathbf{1 0}-5$ & $\mathbf{1 0}-\mathbf{1 0}$ \\
rnd & $9-6$ & $7-3$ & $9-4$ \\
dst & $\mathbf{1 0}-\mathbf{1 0}$ & $8-5$ & $\mathbf{1 0}-8$ \\
\hline \hline iSA & $3-0$ \\
cSA & $5-1$ \\
\hline \multicolumn{4}{c}{ Table III } \\
& ACCURACY OF THE ALGORITHMS.
\end{tabular}

From Table III, we can obtain several interesting conclusions. First, we can note that cooperative schema significantly outperform to non-cooperative ones. In fact, iSA is not able to find the solution to any instance in a robust way. A second important conclusion is that all the variants of our model outperform traditional parallel models for trajectory based methods. This results shows that the exploration scheme induced by our model is more accurate than the other parallel algorithms in the context of this problem.

Analyzing the different variants of the proposed model, it can be seen that the models that make use of some information from the incoming solutions (fitness, distance or shared information) outperform the variants which are based on other features (random or predefined topologies). This is an expected result since the utilization of additional information during the process allows the method to have more elements to guide its search.

In concrete, we can see that cooperation strategies using the best incoming solution or the farthest solution are the best variants. This is a quite surprising result, since these techniques promote very different search behaviours (the bst strategy favors the intensification while the dst scheme promotes the diversification) but both methods get very high-quality solutions. A similar behaviour can be observe when we analyze the different selection method: the bst (which promotes intensification) and inf (which promotes diversification) strategies obtain equivalent results. Although the result are similar, we can notice a clear trend toward the techniques which favor the intensification. In fact, SA_bst_bst, SA_bst_inf and SA_dst_bst are the only ones which can solve all the instances in all the runs.

\section{B. Computational cost}

Now, we focus on the computational cost: numerical performance (number of partial evaluations) and wall-clock time (in seconds). In order to perform a fair comparison, we only consider the algorithms which get similar results. In concrete, we compare SA_bst_bst, SA_bst_inf, SA_dst_bst y SA_dst_inf using the instances which are robustly solved by these methods. In Figure 2, we show the numerical performance (left figure) and the runtime (right figure). Both values are normalized with respect to the value obtained by SA_dst_inf.

From Figure 2, we can distinguish three different behaviours according to the statistical analysis (all the results are statistically different with the exception of SA_X_inf models) . The first behaviour is the presented by SA_dst_bst which is the variant with the highest computational cost. This is expected since the initial solutions selected by dst cooperation strategy are very different and therefore the generated path are longer and it also has to evaluate all the generated solution to find the best solution in the path (bst selection scheme). The second behaviour is the provoked by SA_bst_bst which is the second variant with a higher computational cost. This high cost is due to the utilization of bst selection scheme, in which the variant has to evaluate all the solutions in the path, although in this case the path are usually shorter than in the previous variant (SA_dst_bst). Finally, the last behaviour is the presented by $\mathbf{S} \mathbf{A}_{-} \mathbf{X} \_$inf strategies which need less runtime to find the solution. This is mainly due to the inf selection scheme only need to evaluate a single solution in the path.

Summarizing we can conclude that variants using the best solution in the path obtain the best accurate results but they need more execution time to find these highquality solutions. By contrast, strategies using the shared information between solutions are faster but the solution obtained are slightly worse.

\section{CONClusions ANd Future Works}

In this paper, we have developed a new parallel model for trajectory based methods, which improves the cooperation phase by means of adding path relinking technique. The utilization of this last technique allow to generate a wide set of candidate solutions to continue the search. This set is composed by solutions which include information from the current solution of the subalgorithm and also information from the incoming solution.

The results show that our proposed method is more accurate and efficient than the existing one. We have studied different design alternatives such as the several cooperation schema or different mechanism to select the next solution from the set of solutions generated by path relinking. Each variant has its own advantages and drawbacks. For example, we saw that using the best solution of the path, the algorithm obtains very accurate solution but the computation cost is higher.

As future work, we plan to extend this study to other problems or other trajectory based methods for generalizing the conclusion of this paper. In this paper, we have observed that the evaluation of the point visited by the path generated by $\mathrm{PR}$ is a quite high-consuming process, then we want to analyze different alternatives to perform that process (maybe 


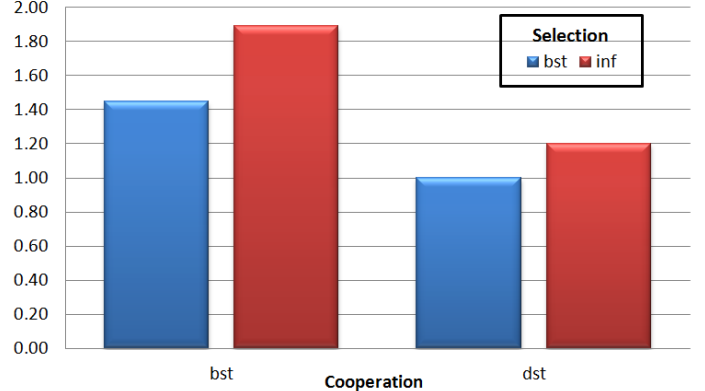

(a)

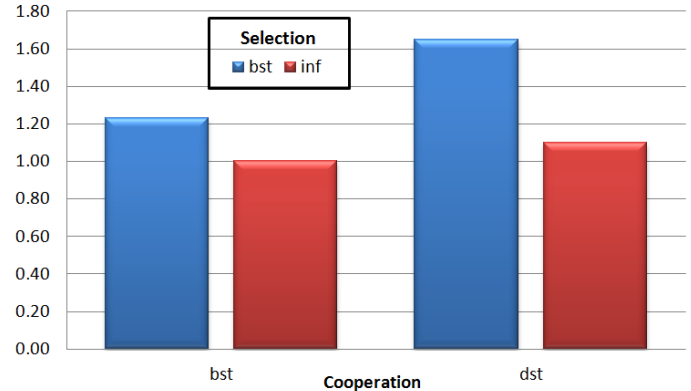

(b)

Figure 2. (a) Numerical performance (number of partial evaluations) and (b) execution time (seconds).

using some theoretical results about the search space) and then speed up the search.

\section{ACKNOWLEDGMENTS}

This research has been partially funded by project number 8.06/5.47.4142 in collaboration with the VSB-Technical University of Ostrava and Universidad de Málaga (Andalucía Tech) UMA/FEDER FC14-TIC36, programa de fortalecimiento de las capacidades de $\mathrm{I}+\mathrm{D}+\mathrm{i}$ en las universidades 2014-2015, de la Consejería e Economía, Innovación, Ciencia y Empleo, cofinanciado por el fondo europeo de desarrollo regional (FEDER) Also, partially funded by the Spanish MINECO project TIN2014-57341$\mathrm{R}$ (http://moveon.lcc.uma.es).

\section{REFERENCES}

[1] E. Alba, editor. Parallel Metaheuristics: A New Class of Algorithms. Wiley, 2005.

[2] E. Alba, G. Luque, and S. Nesmachnow. Parallel metaheuristics: recent advances and new trends. International Transactions in Operational Research, 20(1):1-48, 2013.

[3] W. Bożejko, J. Pempera, and C. Smutnicki. Parallel tabu search algorithm for the hybrid flow shop problem. Computers \& Industrial Engineering, 65(3):466-474, 2013.

[4] Y.-L. Chang, K.-S. Chen, B. Huang, W.-Y. Chang, J. A. Benediktsson, and L. Chang. A parallel simulated annealing approach to band selection for high-dimensional remote sensing images. Selected Topics in Applied Earth Observations and Remote Sensing, IEEE Journal of, 4(3):579-590, 2011.

[5] F. Chicano, G. Luque, and E. Alba. Autocorrelation measures for the quadratiec assignment problem. Applied Mathematics Letters, 25(4):698-705, 2012.

[6] J.-F. Cordeau and M. Maischberger. A parallel iterated tabu search heuristic for vehicle routing problems. Computers \& Operations Research, 39(9):2033-2050, 2012.

[7] M. L. Engle and C. Burks. Artificially generated data sets for testing DNA fragment assembly algorithms. Genomics, 16, 1993.
[8] M. Eskandarpour, S. H. Zegordi, and E. Nikbakhsh. A parallel variable neighborhood search for the multi-objective sustainable post-sales network design problem. International Journal of Production Economics, 145(1):117-131, 2013.

[9] A. Ferreiro, J. García, J. López-Salas, and C. Vázquez. An efficient implementation of parallel simulated annealing algorithm in gpus. Journal of Global Optimization, 57(3):863$890,2013$.

[10] M. Garey and D. Johnson. Computers and Intractability: A Guide to the Theory of NP-Completeness. W. H. Freeman, 1979.

[11] M. Gendreau. Handbook of metaheuristics, volume 146. Springer, 2010.

[12] F. Glover. Tabu Search, part I. ORSA, Journal of Computing, (1):190-206, 1989.

[13] F. Glover, M. Laguna, and R. Martí. Fundamentals of scatter search and path relinking. Control and cybernetics, 39(3):653-684, 2000.

[14] S. Kirkpatrick, C. Gellatt, and M. Vecchi. Optimization by Simulated Annealing. Science, 220(4598):671-680, 1983.

[15] G. Luque, F. Luna, and E. Alba. A new parallel cooperative model for trajectory based metaheuristics. In Distributed Computing and Artificial Intelligence, pages 559567. Springer, 2010.

[16] C. Papadimitriou. The Complexity of Combinatorial Optimization Problems. Master's thesis, Princeton University, 1976.

[17] J. Setubal and J. Meidanis. Introduction to Computational Molecular Biology, chapter 4 - Fragment Assembly of DNA, pages 105-139. University of Campinas, Brazil, 1997.

[18] E.-G. Talbi. Parallel combinatorial optimization, volume 58. John Wiley \& Sons, 2006.

[19] M. Yazdani, M. Amiri, and M. Zandieh. Flexible jobshop scheduling with parallel variable neighborhood search algorithm. Expert Systems with Applications, 37(1):678-687, 2010. 